\title{
ASSEMBLY RELIABILITY MODELLING TECHNOLOGY USING FUNCTION DECOMPOSING AND LSSVM
}

\author{
Yang, D.; Sun, Y.\# \& Wu, K. \\ School of Mechanical Engineering, Nanjing University of Science and Technology, Nanjing, China \\ E-Mail: sunyu@njust.edu.cn ( ${ }^{\sharp}$ Corresponding author)
}

\begin{abstract}
Assembly reliability is an important part of product quality, which greatly affects the reliability level of whole machine. Because of the limitations of FMEA and FTA, they are not suitable for assembly reliability of complex mechanical equipment. A new functional decomposition method for whole machine (FDWM) is proposed for assembly reliability modelling in this paper. Firstly, by decomposing the whole machine into function and connection function of main components, the success tree model for whole machine (STWM) can be established. Secondly, a hybrid method by combining modified grey relation and least squares support vector machine (MGR-LSSVM) is proposed to improve the accuracy of STWM model. Lastly, the proposed method is applied to the assembly reliability analysis for feed mixer. The results show that the FDWM method is feasible for assembly reliability modelling, and the MGR-LSSVM method has a higher accuracy, and is efficient for the reliability analysis.

(Received in November 2019, accepted in March 2020. This paper was with the authors 2 months for 1 revision.)
\end{abstract}

Key Words: Assembly Reliability, Reliability Modelling, STWM Model, Modified Grey Relation, LSSVM

\section{INTRODUCTION}

Assembly process is the terminal link and the most expensive part of the manufacturing cycle, which has the greatest impact on product quality. For a long time, manufacturers of mechanical equipment have been committing to designing research, but overlooking the improvement and optimization of assembly process. Statistics show that assemble process takes up $60 \%$ or more of a production cycle, and causes more about $40 \%$ quality problems [1], which fully demonstrate that the assembly process is an important link that affects product quality. Therefore, in order to improve the reliability of mechanical equipment, the assembly process must be highly valued and studied.

In engineering practice, Failure Mode Effect Analysis (FMEA) [2, 3] and Fault Tree Analysis (FTA) [4, 5] are the most commonly used methods to analyse product quality and system reliability. In the two methods, based on failure analysis, the reliability index of the whole machine can be obtained. However, the two methods fail to combine the functions of the product and motor functions of components, and the failure analysis cannot be accurately identified to specific parts as well. In addition, with the increasingly rapid complex functions and structures of modern electro-mechanical system, the process of product functionformation is becoming multi-levels and multi-factors, which greatly increases the complexity of FMEA and FTA in engineering practices. For these reasons, a new assembly reliability analysis method: Function Decomposing for Whole Machine (FDWM) is proposed for assembly reliability of complex mechanical equipment in this paper. By decomposing the whole machine into functions and connection functions between main components, decomposition process diagram can be built up. By referring the FTA method, the decomposition tree model can be established, which is called success tree for whole machine (STWM) in this paper. Then the assembly reliability of whole machine can be obtained through logical calculation of attribute value of each subitem in STWM. 
For complex mechanical equipment, the STWM model may consists of many items. To reduce modelling complexity and improve evaluation accuracy, many methods have been proposed for many kinds of reliability model, such as the Design for Assembly (DFA) [6], the Assemblability Evaluation Method (AEM) [7], and the Assembly Quality Method (AQM) [8]. These methods have been applied in some fields. However, the DFA and the AEM can't directly assess assembly reliability, and the AQM can only be conducted on the base of a large number of workshop assembly failure data. Furthermore, many scholars are devoted to the study of human reliability in assembly process. But this is just one of many factors that affect the assembly reliability, and cannot be used to evaluate the assembly reliability level comprehensively. In view of the above problems, Suzuki Tatsuya proposed a new method: Assembly Reliability Evaluation Method (AREM) [9, 10]. He divided the influence factors of assembly reliability into two categories: design attributes and production attributes. The former one is related to the characteristics of parts, and it can be obtained by quantitative calculation. The second one is also known as the operating reliability level in the workshop, and it is related to the Basic Fault Rate of Shop (BFRS). By using the questionnaire method, the fault generation coefficient $\left(C_{F G}\right)$, the fault overlook coefficient $\left(C_{F O}\right)$ and the response failure coefficient $\left(C_{R F}\right)$ can be obtained respectively. By multiplying these coefficients with a constant, the BFRS can be calculated. However, the influence degree of these parameters on the assembly reliability is not clear, and cannot provide clear guidance for subsequent assembly process improvement. In recent years, Chen and Jin [11] have proposed a new concept of quality and reliability chain (QR-Chain) effect model to describe this complex relationship between product quality, and manufacturing system (MS) component reliability. This method can be conducted on the basic of system component degradation. In order to achieve the requirement of rapider response and low assembly cost, $\mathrm{Ni}$ et al. proposed the assembly chain decomposition method [4], the 3D precision analysis method [12], the localto-global dimensional error calculation method [13] and the assembly process optimization method [14]. They also discussed the influence of error sources to the global error and the survey on the performance of assembly process optimization, and designed a software tool to calculate the global dimensional error [15]. However, the dimensional error algorithm is not universal. These methods can't calculate the assembly reliability index which fails to combine the reliability assessment and assembly process parameters, and also can't provide guidance for the improvement of assembly process. Furthermore, Xiao et al. [16] proposed a dynamic precision reliability analysis method. But for some engineering machinery, the assembly precision is affected by many factors, and hard to obtain accurately.

The above researches provide valuable method basic and reference value for assembly reliability analysis. But for STWM, the assembly reliability is affected by several factors, the influence law and the influence degree of some factors are hard to certain, and the assembly reliability data belongs to small sample, and only part of its information is known. The assembly process of mechanical equipment can be considered as a grey system. Therefore, the above methods are not suitable, while the grey theory put forward by Deng [17] provides the possibility to solve this problem. In his study, the grey relation analysis (GRA) is used to describe the dynamic development situation and similarity level between multiple factors, which has high accuracy and reliability, when compared with traditional mathematical statistic theory and fuzzy mathematics method. The GRA has been successfully used in many theoretic fields and engineering practices, such as process parameters optimization [18], fault diagnosis [19], decision analysis [20], and so on. In this study, in order to guarantee the accuracy, and determine the relationship between each attribution in STWM model, a hybrid method by combining modified GRA (MGR) and Least Squares Support Vector Machine (LSSVM) is proposed to establish the reliability model of STWM. The LSSVM was proposed by Suykens and Vandewalle [21] in 1999 as a reformulation algorithm for SVM. By using 
least errors as the loss function, LSSVM can reduce the computation time effectively. Since proposed, LSSVM has got a widely application for solving classification and regression problems in various engineering practices, such as engine fault diagnosis [22], parameter forecasting [23], optimum control [24], condition monitoring [25], and other fields [26-28]. Therefore, the combination of GRA and LSSVM can solve the fuzzy problem, and improve the accuracy of reliability modelling effectively. It is of great significance and application prospect to introduce the hybrid MGR-LSSVM method into the field of assembly reliability.

The rest of this paper is arranged as follows. In Section 2, functional decomposition and success tree modelling for whole machine is proposed firstly. Then the modified grey relation -LSSVM model is proposed. Thirdly, the adaptive genetic algorithm is employed for parameters optimization in MGR-LSSVM. In Section 3, the proposed the method is applied to the assembly analysis of feed mixer. The results and discussion are also presented. Lastly, the conclusions are present in Section 4.

\section{METHOD}

\subsection{FDWM method and STWM modelling}

According to the definition of reliability, it can be considered that guaranteeing the reliability of the whole machine is mainly to ensure the function reliability of the machine, while the function of the machine is realized by the motion function of the parts. Therefore, the key of ensuring the reliability can be transformed into guarantee the reliability of the motion function of the parts, while the motion function reliability is carried out by assembly process.

Therefore, by decomposing the function of whole machine into functions connection function of main parts, functional formation process can be carried out, as shown in Fig. 1.

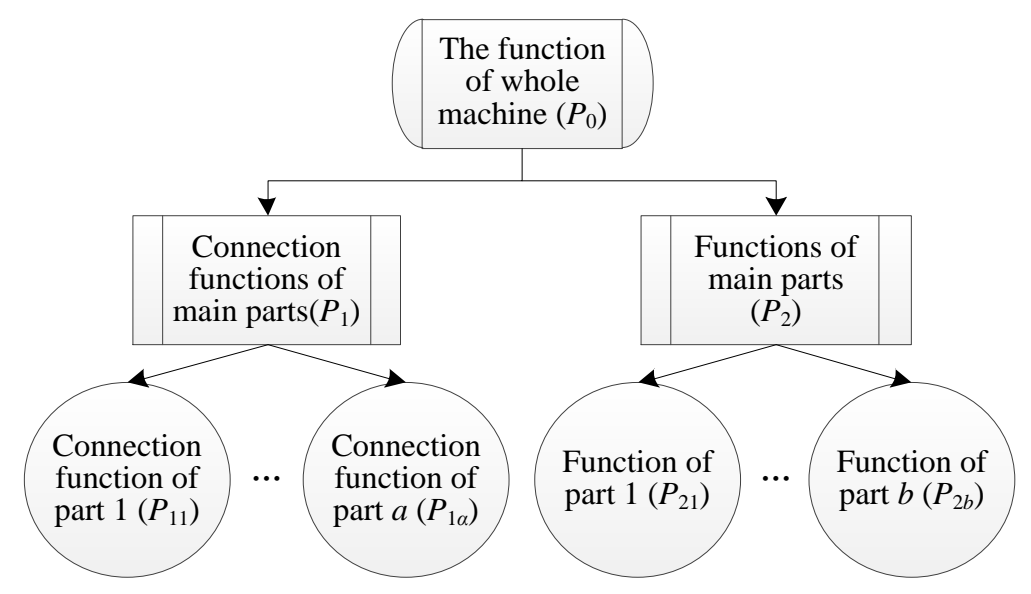

Figure 1: Function decomposing for whole machine.

Based on the process of FDWM, using corresponding symbols to express the events and logical relations in the function decomposition, the decomposition tree can be established. Because this tree is based on the implementation of the function of the whole machine, we call it Success Tree for Whole Machine (STWM) in this paper. In a STWM, the top event is a normal function. The basic events are the lowest level of events required to achieve the function. The intermediate events are other events. It should be noted that the three events here have different meanings with them in FTA. In a STWM, the function of whole machine is on the basic that functions and connection between main components are all reliable, so the relationship between events is "And". By learning from the events and the logical expression in FTA, define symbols in STWM as shown in the Fig. 2. Then the STWM model can be established as shown in Fig. 3. 

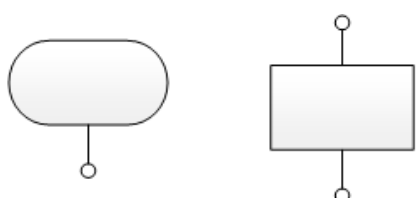

a) top event b) intermediate event
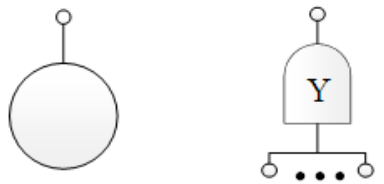

c) basic event

d) AND operation

Figure 2: Symbols in success tree.

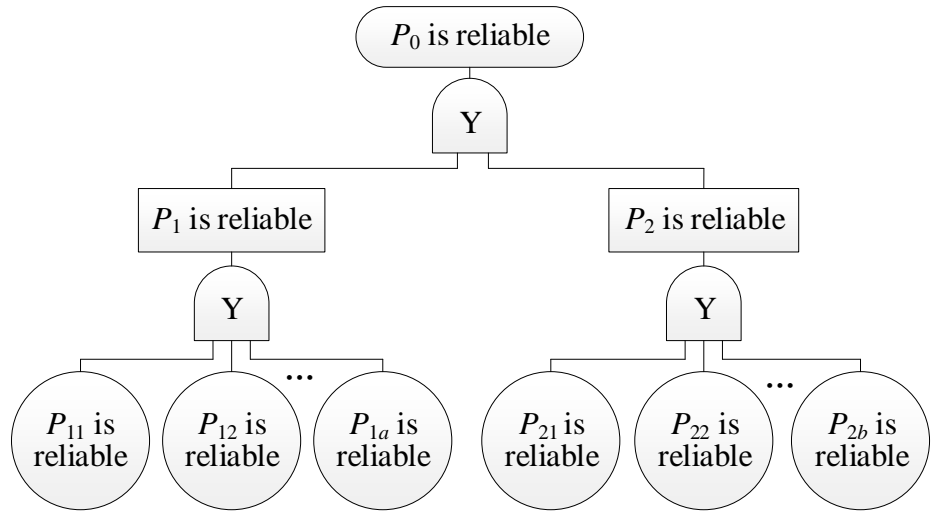

Figure 3: STWEF model.

\subsection{Modified grey relation theory}

In this paper, the assembly reliability data of each functional part is defined as the comparison sequence, $X_{i}=\left(x_{i}(1), x_{i}(2), \ldots, x_{i}(n)\right),(i=1,2, \ldots, m)$, The assembly reliability data of the whole machine is defined as the reference sequence, $X_{0}=\left(x_{0}(1), x_{0}(2), \ldots, x_{0}(n)\right)$. The correlation coefficient of a comparison sequence and the reference sequence at the $j^{\text {th }}$ point is:

$$
\gamma\left(x_{0}(j), x_{i}(j)\right)=\frac{\min _{i} \min _{j}\left|x_{0}(j)-x_{i}(j)\right|+\xi \max _{i} \max _{j}\left|x_{0}(j)-x_{i}(j)\right|}{\left|x_{0}(j)-x_{i}(j)\right|+\xi \max _{i} \max _{j}\left|x_{0}(j)-x_{i}(j)\right|}
$$

In Eq. (1), $\xi$ is the identification coefficient, and $\xi \in(0,1)$. Then the grey relation of the two sequences can be calculated by taking the mean of the coefficients of all corresponding points, as shown in Eq. (2).

$$
R\left(X_{0}, X_{i}\right)=\frac{1}{n} \sum_{j=1}^{n} \gamma\left(x_{0}(j), x_{i}(j)\right)
$$

Though the grey relation analysis has been widely used in various fields, the similarity in shape between two sequences can be obtained by calculating the absolute deviation $\left|x_{0}(j)-x_{i}(j)\right|$. However, the closeness between the two sequences isn't taken in to consideration. The consistency between a comparison sequence and the reference sequence is determined by the two attributes. When the closeness is far away, the similarity in shape cannot compensate for the consistency. Moreover, the value of identification coefficient can reflect the indirect influence degree of each factor on the grey relation. A proper $\xi$, can fully reflect the integrality of the GRA, and eliminate the effect of outliers. Nevertheless, in conventional GRA, the $\xi$ is usually set equal to 0.5 , and cannot be adjusted according to specific application. In order to eliminate the above problems, a new modified grey correlation analysis method is proposed in this paper, as discussed below.

(1) The distance between two sequences is not only related to absolute deviation, but also relative to relative error, which is denoted by $M$, as shown by Eq. (3).

$$
M(j)=\frac{\left|x_{0}(j)-x_{i}(j)\right|}{T(j)}+\frac{\left|x_{0}(j)-x_{i}(j)\right|}{T(j)+\left|x_{0}(j)-x_{i}(j)\right|}
$$

where, $T(j)$ is related to the value of reference sequence. But if the curves of two comparison sequences are on the reference sequence curve symmetry, they have the same closeness. $T(j)$ is defined as:

$$
T(j)= \begin{cases}\sqrt{\left|x_{0}(j)\right|^{\left(1+\operatorname{sgn}\left(x_{0}(j)-x_{i}(j)\right)\right)}} \sqrt{\left|x_{i}(j)\right|^{\left(1-\operatorname{sgn}\left(x_{0}(j)-x_{i}(j)\right)\right)}} & x_{i}(j) \leq x_{0}(j) \\ \sqrt{\left|x_{0}(j)\right|^{\left(1+\operatorname{sgn}\left(x_{i}(j)-x_{0}(j)\right)\right)}} \sqrt{\left|x_{i}(j)\right|^{\left(1-\operatorname{sgn}\left(x_{i}(j)-x_{0}(j)\right)\right)}} & x_{i}(j)>x_{0}(j)\end{cases}
$$


In grey relational analysis, different requirements for the similarity and the adjacent degree of the comparison sequence and reference sequence can cause different analysis result. Therefore, two variables $\alpha$ and $\beta$ are employed to reflect the similarity and the adjacent degree, respectively. Thus, the traditional GRA can be modified as shown in Eq. (5).

$$
\gamma^{\prime}\left(x_{0}(j), x_{i}(j)\right)=\left[\gamma\left(x_{0}(j), x_{i}(j)\right)\right]^{\alpha}[\exp (-M(j))]^{\beta}
$$

where $\alpha+\beta=1$, and $\alpha, \beta \in(0,1)$.

(2) The GRA can express the system's entirety by calculated the maximum value of absolute deviation. The constant coefficient $\xi$ should be able to reflect the indirect effects of each factor on the relation degree. Meanwhile, it should have anti-interference ability, namely can reduce the error effect of the outliers in the observed sequence to the whole relation space. To obtain a better solution, the value of the identification coefficient $\xi$ is improved, as shown below.

Definition 1: $\eta_{\Delta}$ is a dynamic coefficient:

$$
\eta_{\Delta}=\Delta_{v} / \Delta_{\max }
$$

where:

$$
\begin{gathered}
\Delta_{v}=\frac{1}{n m} \sum_{i=1}^{n} \sum_{j=1}^{m}\left|x_{0}(j)-x_{i}(j)\right| \\
\Delta_{\max }=\max _{i} \max _{j}\left|x_{0}(j)-x_{i}(j)\right|
\end{gathered}
$$

The value of $\xi$ can be determined by the following equation:

$$
\xi= \begin{cases}{\left[\eta_{\Delta}, 1.5 \eta_{\Delta}\right]} & \Delta_{\max }>3 \Delta_{v} \\ {\left[1.5 \eta_{\Delta}, 2 \eta_{\Delta}\right]} & 2 \Delta_{v}<\Delta_{\max } \leq 3 \Delta_{v} \\ (0,1] & 0 \leq \Delta_{\max } \leq 2 \Delta_{v}\end{cases}
$$

(3) In GRA, the absolute deviation is calculated point-by-point, and then be used to calculated the weighted average. This method can easily to cause the missing of locally associated information. However, as for reliability modelling, the historical data of different times do not always have the same influence on the present situation. In general, the closer the data is, the greater the influence is. Therefore, a weighted value is adopted for the historical data of different times by using fuzzy weighting method, as discussed below.

Definition 2: the relative importance of two reliability data at time $t_{p}$ and $t_{q}$ is denoted by $f_{p q}$, which can be determined by Eq. (10).

$$
f_{p q}= \begin{cases}1, & \text { If } t_{p} \text { is as important as } t_{q} \\ p-q+1, & \text { If } t_{p} \text { is more important than } t_{q} \\ 1 /(q-p+1), & \text { If } t_{q} \text { is more important than } t_{p}\end{cases}
$$

Then the fuzzy judgment matrix is established, denoted by $\boldsymbol{F}=\left[f_{p q}\right]_{n \times n}$. Transforming $\boldsymbol{F}$ by using Eq. (11), the fuzzy consistent matrix $\boldsymbol{R}$ can be established.

$$
r_{p q}=\frac{1}{2}\left(\log _{M}\left(f_{p q}\right)+1\right)
$$

where, $[1 / M, M]$ is the scale of matrix $\boldsymbol{F}$. When $w_{1}, w_{2}, \ldots, w_{\mathrm{n}}$ are the weighted value of time $t_{1}, t_{2}, \ldots, t_{n}$, respectively, the following equations can be established according to the property of the fuzzy consistent matrix $\boldsymbol{R}$.

$$
\begin{gathered}
r_{p q}=0.5+a\left(w_{p}-w_{q}\right) \\
w_{j}=\frac{1}{n}-\frac{1}{2 a}+\frac{1}{n a} \sum_{k=1}^{n} r_{j k}
\end{gathered}
$$

Taking the weighted relation into consideration, then the GRA can be revised, 


$$
R^{\prime}\left(X_{0}, X_{i}\right)=\sum_{j=1}^{n} w(j) \rho\left(x_{0}(j), x_{i}(j)\right)
$$

In conclusion, taking the relative error $M$, the identification coefficient $\xi$, and the weighted relation $w$, the modified GRA is obtained as shown in Eq. (15).

$$
R\left(X_{0}, X_{i}\right)=\sum_{j=1}^{n}\left[w(j)\left[\gamma\left(x_{0}(j), x_{i}(j)\right)\right]^{\alpha}[\exp (-M(j))]^{\beta}\right]
$$

By comparing the GR value of each sequence, the high relevance sequences can be determined.

\subsection{LSSVM algorithm}

On the basic of GRA in Section 2.2, the modelling of relationship between functional parts and the whole machine can be established by Least Squares Support Vector Machine. In MGR-LSSVM model, the input parameter is the large-relation sequence, denoted by $x_{i}$. The output parameter is assembly reliability of the whole machine, denoted by $y_{i}$. Using the structural risk minimization (SRM) principle, the optimization function of LSSVM is:

$$
\min _{\omega, b, e} J(\omega, e)=\frac{1}{2} \omega^{\mathrm{T}} \omega+\gamma \frac{1}{2} \sum_{i=1}^{l} e_{i}^{2}
$$

The constraint conditions are:

$$
y_{i}=\varphi\left(x_{i}\right) \times \omega+b+e_{i}, \quad i=1,2, \ldots, l
$$

where, $\omega$ is weight vector, $\gamma$ is the regularization parameter which balances the model's complexity and the training error, $e_{i}$ is error vector, $b$ is offset. The Lagrange function of the objective function $J$ is:

$$
L(\omega, b, e, \alpha)=J(\omega, e)-\sum_{i=1}^{l} \alpha_{i}\left(\omega^{\mathrm{T}} \varphi\left(x_{i}\right)+b+e_{i}-y_{i}\right)
$$

where $\alpha_{i}$ is the Lagrange multiplier of the $i^{\text {th }}$ sample. According to the Karush-Kuhn-Tucker (KKT) conditions, calculate the first-order derivative:

$$
\frac{\partial L}{\partial \omega}=0, \frac{\partial L}{\partial b}=0, \frac{\partial L}{\partial e}=0, \frac{\partial L}{\partial a}=0
$$

then by eliminating variable $\omega$ and $e_{i}$, the simplified equation can be obtained:

$$
\begin{gathered}
{\left[\begin{array}{cc}
0 & A \\
A^{\mathrm{T}} & \Omega+\frac{1}{\mu} I
\end{array}\right]\left[\begin{array}{c}
b \\
a^{\mathrm{T}}
\end{array}\right]=\left[\begin{array}{c}
0 \\
y^{\mathrm{T}}
\end{array}\right]} \\
\Omega_{i j}=K\left(x_{i}, x_{j}\right)=\varphi\left(x_{i}\right) \varphi\left(x_{j}\right)
\end{gathered}
$$

where $A$ is a $l$-dimensional unit row vector, $I$ is a unit matrix with $l \cdot l$ order, $K\left(x_{i}, x_{j}\right)$ is a symmetric function which satisfied the Mercer theory, $\alpha$ is a $l$-dimensional vector, $\alpha=\left[\alpha_{1}, \alpha_{2}, \ldots, \alpha_{l}\right], y$ is a $l$-dimensional output vector, $y=\left[y_{1}, y_{2}, \ldots, y_{l}\right], \boldsymbol{\Omega}$ is a $l \cdot l$ symmetric matrix. By solving Eq. (20), model parameters $\alpha$ and $b$ can be obtained.

The radial basic function (RBF), as shown in Eq. (22), is the most commonly used kernel function because of its significant regression characteristic.

$$
K\left(x, x_{i}\right)=\exp \left(-\left\|x-x_{i}\right\|^{2} / \sigma^{2}\right)
$$

where $\sigma$ is the kernel parameter. As a consequence, the LSSVM model becomes:

$$
y(x)=\sum_{i=1}^{n} \alpha_{i} K\left(x_{i}, x_{j}\right)+b
$$




\subsection{Parameters optimization for LSSVM}

In general, the regularization parameter $\gamma$ and the kernel parameter $\sigma$ in LSSVM model can directly affect the generalization ability and approximation of the MGR-LSSVM model. To optimize the two parameters, and improve the accuracy of LSSVM, the genetic algorithm (GA) provides a good solution [29, 30]. But because of the problem of premature convergence and slow evolution of GA [31, 32], an adaptive genetic algorithm (AGA) is adopted in this paper. In the AGA, the crossover rate and the mutation rate can be changed with the colony fitness degree of each generation automatically [33, 34]. When the individual fitness converges to the local optimum, the crossover rate and the mutation rate can be increased. Otherwise when the individual fitness scattered, the crossover rate and the mutation rate should be decreased. Meanwhile, the individual which is upon the average fitness value, should be protected to enter the next generation, otherwise it can be eliminated. The crossover rate $P_{c}$ and the mutation rate $P_{m}$ can be obtained by Eqs. (24) and (25), respectively.

$$
\begin{gathered}
P_{c}=\left\{\begin{array}{cc}
P_{1}-\frac{\left(P_{1}-P_{2}\right)\left(f-f_{\text {avg }}\right)}{f_{\text {max }}-f_{\text {avg }}}, & f \geq f_{\text {avg }} \\
P_{1}, & f<f_{\text {avg }}
\end{array}\right. \\
P_{m}=\left\{\begin{array}{cc}
P_{3}-\frac{\left(P_{3}-P_{4}\right)\left(f_{\text {max }}-f\right)}{f_{\text {max }}-f_{\text {avg }}}, & f^{\prime} \geq f_{\text {avg }} \\
P_{3}, & f^{\prime}<f_{\text {avg }}
\end{array}\right.
\end{gathered}
$$

where $f_{\max }$ is the maximum fitness value in the current generation, $f_{\text {avg }}$ is the average fitness value, $f$ is the larger fitness value of the two crossover-individuals, $f$ ' is the fitness value of the mutation individual, $P_{1}, P_{2}, P_{3}$ and $P_{4}$ are constants.

\section{RESULTS AND DISCUSSION}

\subsection{STWM model of feed mixer}

In this paper, a two-axis horizontal feed mixer is taken as the research object (SLHSJ7, Jiangsu FAMSUN Intelligent Technology CO. LTD, China). By using the decomposition method proposed in Section 2.1, the STWM model is established.

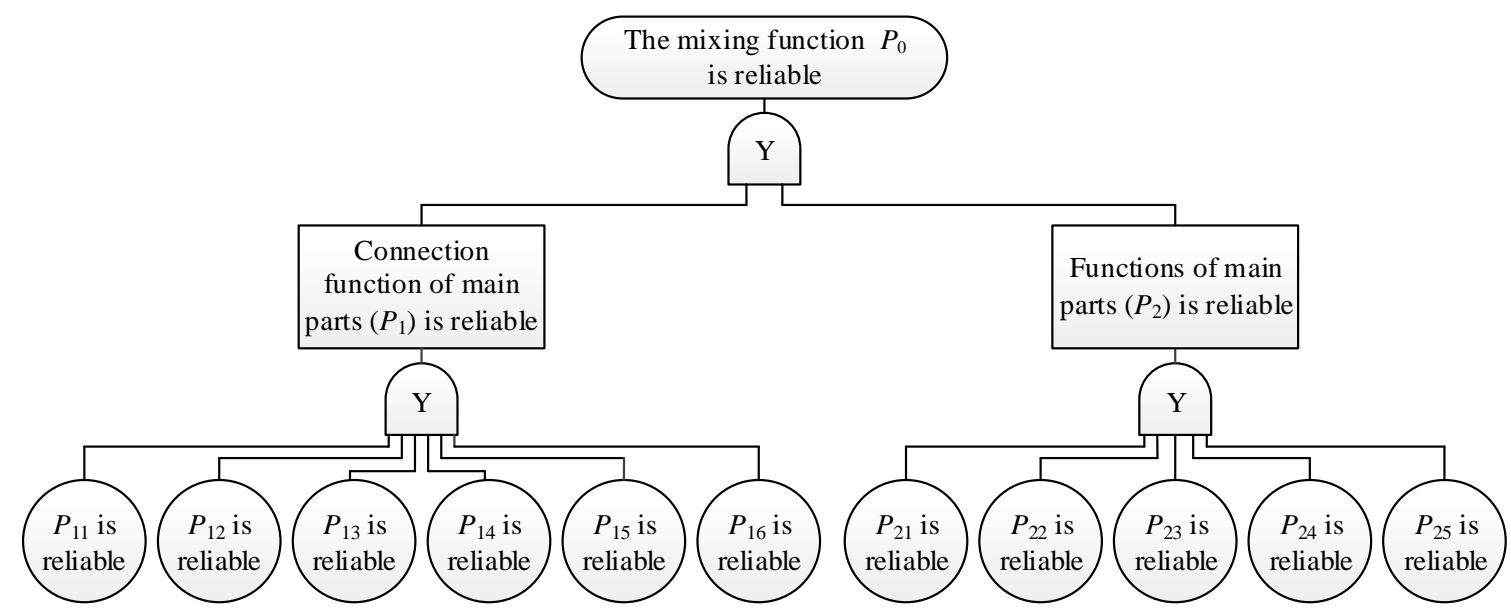

Figure 4: The STWM model of feed mixer.

As shown in Fig. $4, P_{11}$ is the connection of the spindle and the rack, $P_{12}$ is the connection of the discharge door and the rack, $P_{13}$ is the connection of the link mechanism and the rack, $P_{14}$ is the connection of the oil pipeline and the rack, $P_{15}$ is the connection of the cleaning 
door and the rack, $P_{16}$ is the connection of the gearbox and the rack. $P_{21}, P_{22}, P_{23}, P_{24}$, and $P_{25}$ are the functions of the spindle, the discharge door, the link mechanism, the oil pipeline, and the chain, respectively. Based on the STMW model, an assembly reliability data was collected, as shown in Table I. The assembly reliability sequence of the whole machine can also be obtained, $X_{0}=[0.7098,0.7570,0.7305,0.8056,0.6627,0.7634]$.

Table I: Assembly reliability data from 2013 to 2018.

\begin{tabular}{|c|c|c|c|c|c|c|c|}
\hline Sequence & Function & $\mathbf{2 0 1 3}$ & $\mathbf{2 0 1 4}$ & $\mathbf{2 0 1 5}$ & $\mathbf{2 0 1 6}$ & $\mathbf{2 0 1 7}$ & $\mathbf{2 0 1 8}$ \\
\hline$X_{1}$ & $\mathrm{P}_{11}$ & 0.9655 & 0.9675 & 0.9846 & 0.9746 & 0.9363 & 0.9681 \\
\hline$X_{2}$ & $\mathrm{P}_{12}$ & 0.9784 & 0.9711 & 0.9653 & 0.9783 & 0.9804 & 0.9787 \\
\hline$X_{3}$ & $\mathrm{P}_{13}$ & 0.9526 & 0.9747 & 0.9653 & 0.9783 & 0.9412 & 0.9752 \\
\hline$X_{4}$ & $\mathrm{P}_{14}$ & 0.9914 & 0.9783 & 0.9884 & 0.9674 & 0.9804 & 0.9823 \\
\hline$X_{5}$ & $\mathrm{P}_{15}$ & 0.9914 & 0.9819 & 0.9691 & 0.9891 & 0.9755 & 0.9894 \\
\hline$X_{6}$ & $\mathrm{P}_{16}$ & 0.9612 & 0.9567 & 0.9691 & 0.9819 & 0.9657 & 0.9645 \\
\hline$X_{7}$ & $\mathrm{P}_{21}$ & 0.9526 & 0.9675 & 0.9537 & 0.9746 & 0.9608 & 0.9681 \\
\hline$X_{8}$ & $\mathrm{P}_{22}$ & 0.9741 & 0.9856 & 0.9730 & 0.9819 & 0.9902 & 0.9894 \\
\hline$X_{9}$ & $\mathrm{P}_{23}$ & 0.9526 & 0.9892 & 0.9614 & 0.9855 & 0.9608 & 0.9539 \\
\hline$X_{10}$ & $\mathrm{P}_{24}$ & 0.9871 & 0.9856 & 0.9884 & 0.9928 & 0.9706 & 0.9894 \\
\hline$X_{11}$ & $\mathrm{P}_{25}$ & 0.9569 & 0.9675 & 0.973 & 0.9819 & 0.9363 & 0.9752 \\
\hline
\end{tabular}

\subsection{Results and discussion}

Using the MGR method given by Eq. (5), the relation coefficient of the reference sequence $X_{i}$ and the compare sequence $X_{0}$ can be obtained. The values of model parameters are: $\alpha=0.7$, $\beta=0.3$. Using Eqs. (10) to (13), the weighted values are obtained: $w=(0.0443,0.0905$, $0.1409,0.1925,0.2428,0.2891)$. According to Eq. (15), the grey relation analysis of whole machine assembly reliability and functional parts is carried out, $R=(0.7987,0.7839,0.7967$, $0.7801,0.7753,0.7943,0.7982,0.7734,0.7968,0.7720,0.7963)$. Therefore, the first four parts $\left\{X_{1}, X_{7}, X_{9}, X_{3}\right\}$ can be considered as the high-correlation sequence. Similarly, the traditional grey relation method shown in Eq. (1) is also conducted for the reliability data, the high relevance sequence can be obtained, $\left\{X_{7}, X_{3}, X_{6}, X_{11}\right\}$, which shows that there is a big difference between the two high-correlation sequences.

Based on the result of GR, the LSSVM model of assemble reliability is established, and the adaptive genetic algorithm is employed for the parameter optimization. The MGALSSVM model is trained on the assemble data from 2013 to 2017, and is then tested on the remaining data of 2018. The parameters of MGA-LSSVM model are shown in Table II.

Table II: Parameters of GA-LSSVM model.

\begin{tabular}{|c|c|}
\hline Parameter & Value \\
\hline Number of inputs & 3 \\
\hline Kernel function & RBF \\
\hline Population size & 20 \\
\hline Maximum iteration & 200 \\
\hline$P_{1}$ & 0.9 \\
\hline$P_{2}$ & 0.3 \\
\hline$P_{3}$ & 0.1 \\
\hline$P_{4}$ & 0.001 \\
\hline
\end{tabular}

In the AGA method, the fitness function is used to evaluate the quality of individuals, which has important influence on the selection, crossover and mutation operation of genetic 
algorithm. In this study, to evaluation the accuracy of the LSSVM model, root mean square error $(R M S E)$ is chosen as the fitness function as the following equation shows.

$$
R M S E=\sqrt{\frac{1}{n} \sum_{i=1}^{n}\left(y_{i}-y_{i p}\right)^{2}}
$$

where $n$ is the number of the data sample, $y_{i}$ and $y_{i p}$ are the actual value and the prediction value, respectively. Furthermore, relative root mean square error (RRMSE), and coefficient of determination $\left(R^{2}\right)$, mean absolute percentage error $(M A P E)$ are also used to evaluate the performance of the model, as sown below.

$$
\begin{gathered}
\text { RRMSE }=\left(\sqrt{\frac{1}{n} \sum_{i=1}^{n}\left(y_{i}-y_{i p}\right)^{2}} / \frac{1}{n} \sum_{i=1}^{n} y_{i}\right) \times 100 \% \\
R^{2}=1-\sum_{i=1}^{n}\left(y_{i}-y_{i p}\right)^{2} / \sum_{i=1}^{n} y_{i}^{2} \\
M A P E=\frac{1}{n} \sum_{i=1}^{n}\left|\left(y_{i}-y_{i p}\right) / y_{i}\right| \times 100 \%
\end{gathered}
$$

Normally, a lower RMSE, MAPE, and a higher $R^{2}$, represent a higher accuracy of the GALSSVM model. Moreover, the RRMSE values can be construed as follows: unexceptionable if $R R M S E<5 \%$, fine if $5 \%<R R M S E<20 \%$, and poor if $R R M S E>20 \%$.

The performance of the AGA evolution process is shown in Fig. 5. The statistical error of the optimized result is shown Table III. In order to further evaluate the performance of the MGR-AGA-LSSVM model proposed in this paper, the GR-GA-LSSVM model with traditional grey relation algorithm and traditional genetic algorithm is also studied. The two models are encoded in MATLAB 2016a, and the performed computer is equipped with 3.6 GHz CPU and 8 GB RAM.

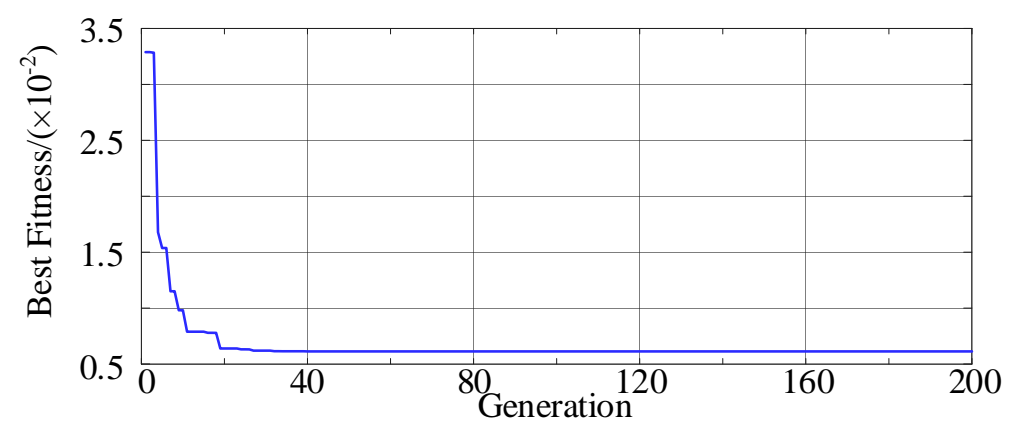

Figure 5: Performance of the AGA evolution process.

Table III: The statistical error of the optimized result.

\begin{tabular}{|c|c|c|c|c|}
\hline Model & $\boldsymbol{R M S E}$ & $\boldsymbol{R R M S E}$ & $\boldsymbol{R}^{\mathbf{2}}$ & $\boldsymbol{M A P E}$ \\
\hline MGR-AGA-LSSVM & $6.094 \mathrm{e}-3$ & $8.312 \mathrm{e}-3$ & 0.9999 & $7.383 \mathrm{e}-3$ \\
\hline GR-GA-LSSVM & $1.210 \mathrm{e}-2$ & $1.663 \mathrm{e}-2$ & 0.9997 & $1.604 \mathrm{e}-2$ \\
\hline
\end{tabular}

From Table III, it can be seen that the RMSE, RRMSE, $R^{2}, M A P E$ obtained by MGRAGA-LSSVM model are smaller than by the GR-GA-LSSVM model. The result shows fully convincingly that the MGR-AGA-LSSVM model has a higher accuracy. In addition, the real assembly reliability value and the LSSVM prediction value from 2013 to 2018 is given in Table IV and Fig. 6. There, it is illustrated that the prediction value obtained by the MGRAGA-LSSVM model has a smaller error between the real assembly reliability values. The MGR-AGA-LSSVM prediction error of year 2018 is $0.5764 \%$, while the GR-GA-LSSVM prediction error is $1.952 \%$. These results demonstrate that the new Function Decomposing for 
Whole Machine is feasible for assembly reliability analysis, and the MGR-AGA-LSSVM model is efficient for the reliability model, and has a high accuracy.

Table IV: Comparison of the real assembly reliability and the prediction assembly reliability.

\begin{tabular}{|c|c|c|c|}
\hline Year & Real value & MGR-AGA-LSSVM & GR-GA-LSSVM \\
\hline 2013 & 0.7098 & 0.7122 & 0.7031 \\
\hline 2014 & 0.7570 & 0.7548 & 0.7703 \\
\hline 2015 & 0.7305 & 0.7385 & 0.7184 \\
\hline 2016 & 0.8056 & 0.7968 & 0.8137 \\
\hline 2017 & 0.6627 & 0.6685 & 0.6803 \\
\hline 2018 & 0.7634 & 0.7590 & 0.7783 \\
\hline
\end{tabular}

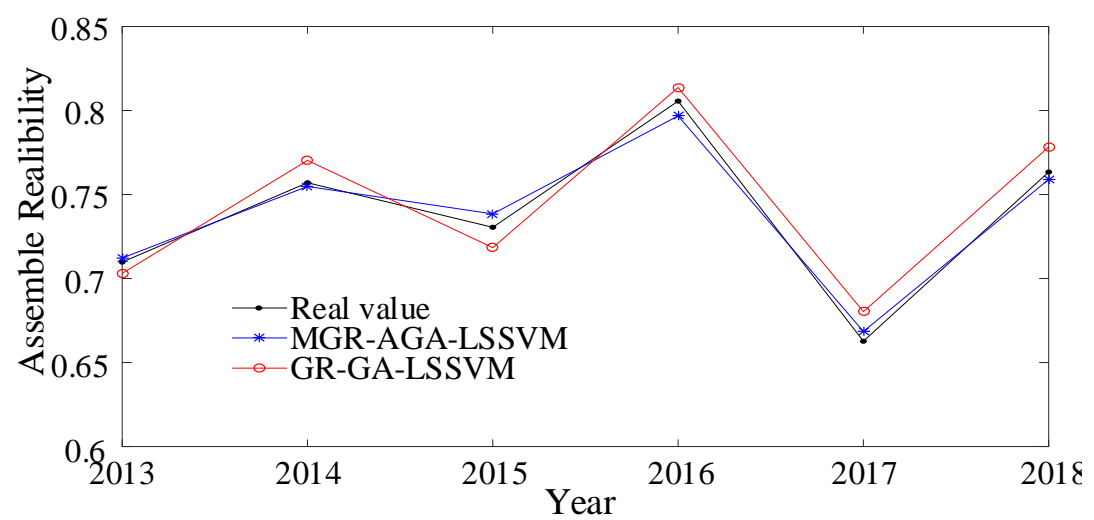

Figure 6: Comparison of the real assembly reliability and the prediction assembly reliability.

\section{CONCLUSIONS}

This paper proposes a new assembly reliability modelling method: Functional Decomposition Method for Whole Machine (FDWM). By decomposing the whole machine into functions and connection function of main components, the success tree model for whole machine (STWM) can be established. With the scientific problem illustrated, the MGR-AGA-LSSVM are put forward to guarantee the effectiveness of the STWM model.

A case of feed mixer is studied. In comparison to the GR-GA-LSSVM method, the results illustrate that the MGR-AGA-LSSVM model is more efficient, which can obtain a smaller $R M S E, R R M S E, M A P E$, a bigger $R^{2}$, and get a higher prediction accuracy.

The FDWM method has been proved to be effective and feasible for assembly reliability modelling. This method has an important application prospect, which can not only be used to feed mixer, but also many other complex machines.

\section{ACKNOWLEDGEMENT}

This work was supported by the Funds for Transformation of Scientific and Technological Achievements in Jiangsu Province, China (BA2017081).

\section{REFERENCES}

[1] Li, D.; Zhang, G.; Li, M.; Lou, J.; Zhao, H. (2015). Assembly reliability modeling technology based on meta-action, Procedia CIRP, Vol. 27, 207-215, doi:10.1016/j.procir.2015.04.068

[2] Liu, H.-C.; Li, P.; You, J.-X.; Chen, T.-Z. (2015). A novel approach for FMEA: combination of interval 2-tuple linguistic variables and gray relational analysis, Quality and Reliability Engineering International, Vol. 31, No. 5, 761-772, doi:10.1002/qre.1633 
[3] Wang, X.; Zhang, Y.; Shen, G. (2016). An improved FMECA for feed system of CNC machining center based on ICR and DEMATEL method, The International Journal of Advanced Manufacturing Technology, Vol. 83, No. 1-4, 43-54, doi:10.1007/s00170-015-7551-y

[4] Ni, J.; Tang, W.; Xing, Y. (2013). A simple algebra for fault tree analysis of static and dynamic systems, IEEE Transactions on Reliability, Vol. 62, No. 4, 846-861, doi:10.1109/ $\underline{\text { TR.2013.2285035 }}$

[5] Yan, R.; Jackson, L. M.; Dunnett, S. J. (2017). Automated guided vehicle mission reliability modelling using a combined fault tree and Petri net approach, The International Journal of Advanced Manufacturing Technology, Vol. 92, No. 5-8, 1825-1837, doi:10.1007/s00170-017$\underline{0175-7}$

[6] Boothroyd, G.; Alting, L. (1992). Design for assembly and disassembly, CIRP Annals, Vol. 41, No. 2, 625-636

[7] Ohashi, T.; Iwata, M.; Arimoto, S.; Miyakawa, S. (2002). Extended assemblability evaluation method (AEM), JSME International Journal Series C Mechanical Systems, Machine Elements and Manufacturing, Vol. 45, No. 2, 567-574, doi:10.1299/jsmec.45.567

[8] Shibata, H.; Cheldelin, B.; Ishii, K. (2003). Assembly quality methodology: a new method for evaluating assembly complexity in globally distributed manufacturing, Proceedings of the 2003 ASME International Mechanical Engineering Congress and Exposition, 335-344, doi:10.1115/IMECE2003-42415

[9] Suzuki, T.; Ohashi, T.; Asano, M. (2003). Assembly reliability evaluation method (AREM), CIRP Annals, Vol. 52, No. 1, 9-12, doi:10.1016/S0007-8506(07)60518-6

[10] Suzuki, T.; Ohashi, T.; Asano, M.; Arai, T. (2004). AREM shop evaluation method, CIRP Annals, Vol. 53, No. 1, 43-46, doi:10.1016/S0007-8506(07)60641-6

[11] Chen, Y.; Jin, J. (2005). Quality-reliability chain modeling for system-reliability analysis of complex manufacturing processes, IEEE Transactions on Reliability, Vol. 54, No. 3, 475-488, doi:10.1109/TR.2005.853441

[12] Ni, J.; Tang, W.; Xing, Y. (2014). Three-dimensional precision analysis with rigid and compliant motions for sheet metal assembly, The International Journal of Advanced Manufacturing Technology, Vol. 73, No. 5-8, 805-819, doi:10.1007/s00170-014-5832-5

[13] Ni, J.; Tang, W.; Xing, Y.; Ben, K.; Li, M. (2016). A local-to-global dimensional error calculation framework for the riveted assembly using finite-element analysis, Journal of Manufacturing Science and Engineering, Vol. 138, No. 3, Paper 031004, 13 pages, doi: $10.1115 / 1.4031101$

[14] Ni, J.; Tang, W. C.; Xing, Y. (2015). Assembly process optimization for reducing the dimensional error of antenna assembly with abundant rivets, Journal of Intelligent Manufacturing, Vol. 29, No. 1, 245-258, doi:10.1007/s10845-015-1105-x

[15] Ni, J.; Tang, W. C.; Xing, Y. (2017). Performance of reducing the dimensional error of an assembly by the rivet upsetting direction optimization, Proceedings of the Institution of Mechanical Engineers, Part B: Journal of Engineering Manufacture, Vol. 231, No. 12, 2133 2144, doi: $10.1177 / 0954405415625918$

[16] Xiao, M.; Geng, G.; Li, G.; Li, H.; Ma, R. (2017). Analysis on dynamic precision reliability of high-speed precision press based on Monte Carlo method, Nonlinear Dynamics, Vol. 90, No. 4, 2979-2988, doi:10.1007/s11071-017-3857-7

[17] Deng, J.-L. (1982). Control problems of grey systems, Systems \& Control Letters, Vol. 1, No. 5, 288-294, doi:10.1016/S0167-6911(82)80025-X

[18] Zhou, J.; Ren, J.; Tian, W. (2017). Grey-RBF-FA method to optimize surface integrity for inclined end milling Inconel 718, The International Journal of Advanced Manufacturing Technology, Vol. 91, No. 9-12, 2975-2993, doi:10.1007/s00170-016-9897-1

[19] Khoualdia, T.; Handjadj, A. E.; Bouacha, K.; Abdeslam, D. O. (2017). Multi-objective optimization of ANN fault diagnosis model for rotating machinery using grey rational analysis in Taguchi method, The International Journal of Advanced Manufacturing Technology, Vol. 89, No. 9-12, 3009-3020, doi:10.1007/s00170-016-9278-9

[20] Chen, J.-H.; Hsu, S.-C.; Wang, R.; Chou, H.-A. (2017). Improving hedging decisions for financial risks of construction material suppliers using grey system theory, Journal of 
Management in Engineering, Vol. 33, No. 4, Paper 040170164, 10 pages, doi:10.1061/ (ASCE)ME.1943-5479.0000531

[21] Suykens, J. A. K.; Vandewalle, J. (1999). Least squares support vector machine classifiers, Neural Processing Letters, Vol. 9, No. 3, 293-300, doi:10.1023/A:1018628609742

[22] Yao, D.; Yang, J.; Li, X.; Zhao, C. (2016). A hybrid approach for fault diagnosis of railway rolling bearings using STWD-EMD-GA-LSSVM, Mathematical Problems in Engineering, Vol. 2016, Paper 8702970, 7 pages, doi:10.1155/2016/8702970

[23] Niu, D.; Dai, S. (2017). A short-term load forecasting model with a modified particle swarm optimization algorithm and least squares support vector machine based on the denoising method of empirical mode decomposition and grey relational analysis, Energies, Vol. 10, No. 3, Paper 408, 20 pages, doi:10.3390/en10030408

[24] Fei, C.-W.; Bai, G.-C.; Tang, W.-Z.; Choy, Y. (2015). Optimum control for nonlinear dynamic radial deformation of turbine casing with time-varying LSSVM, Advances in Materials Science and Engineering, Vol. 2015, Paper 680406, 9 pages, doi:10.1155/2015/680406

[25] Lin, X.; Zhou, B.; Zhu, L. (2017). Sequential spindle current-based tool condition monitoring with support vector classifier for milling process, The International Journal of Advanced Manufacturing Technology, Vol. 92, No. 9-12, 3319-3328, doi:10.1007/s00170-017-0396-9

[26] Zhao, H.; Ru, Z.; Chang, X.; Yin, S.; Li, S. (2014). Reliability analysis of tunnel using least square support vector machine, Tunnelling and Underground Space Technology, Vol. 41, 14-23, doi:10.1016/j.tust.2013.11.004

[27] Khatibinia, M.; Fadaee, M. J.; Salajegheh, J.; Salajegheh, E. (2013). Seismic reliability assessment of RC structures including soil-structure interaction using wavelet weighted least squares support vector machine, Reliability Engineering \& System Safety, Vol. 110, 22-33, doi:10.1016/j.ress.2012.09.006

[28] He, P. (2018). Optimization and simulation of remanufacturing production scheduling under uncertainties, International Journal of Simulation Modelling, Vol. 17, No. 4, 734-743, doi:10.2507/IJSIMM17(4)CO20

[29] Chen, C.; Sun, Y.; Ni, J. (2018). Optimization of flexible fixture layout using N-M principle, The International Journal of Advanced Manufacturing Technology, Vol. 96, No. 9-12, 4303-4311, doi:10.1007/s00170-018-1907-z

[30] Wang, C.; Fu, G.; Zhang, D.; Wang, H.; Zhao, J. (2019). Genetic algorithm-based variable value control method for solving the ground target attacking weapon-target allocation problem, Mathematical Problems in Engineering, Vol. 2019, Paper 6761073, 9 pages, doi: $10.1155 / 2019 / 6761073$

[31] Wang, W. M.; Li, D. B.; He, F.; Tong, Y. F. (2018). Modelling and optimization for a selective assembly process of parts with non-normal distribution, International Journal of Simulation Modelling, Vol. 17, No. 1, 133-146, doi:10.2507/IJSIMM17(1)CO1

[32] Yang, M. S.; Ba, L.; Liu, Y.; Zheng, H. Y.; Yan, J. T.; Gao, X. Q.; Xiao, J. M. (2019). An improved genetic simulated annealing algorithm for stochastic two-sided assembly line balancing problem, International Journal of Simulation Modelling, Vol. 18, No. 1, 175-186, doi:10.2507/IJSIMM18(1)CO4

[33] Yang, D.; Sun, Y.; Wu, K. (2020). Research on CEEMD-AGA denoising method and its application in feed mixer, Mathematical Problems in Engineering, Vol. 2020, Paper 9873268, 9 pages, doi: $10.1155 / 2020 / 9873268$

[34] Xiao, M.; Wen, K.; Zhang, C.; Zhao, X.; Wei, W.; Wu, D. (2018). Research on fault feature extraction method of rolling bearing based on NMD and wavelet threshold denoising, Shock and Vibration, Vol. 2018, Paper 9495265, 11 pages, doi: $\underline{10.1155 / 2018 / 9495265}$ 